\title{
On $\mathrm{I}_{2}$-Cauchy Double Sequences in p-Adic Linear 2-Normed Spaces
}

\author{
B. Surender Reddy ${ }^{1}$, D. Shankaraiah ${ }^{2}$ \\ ${ }^{1}$ Department of Mathematics, University College of Science, Saifabad, Osmania University, Hyderabad-500 \\ 004, AP, INDIA \\ ${ }^{2}$ Department of Mathematics, CVR College of Engineering, Ibrahimpatnam, Ranga Reddy District-501510, AP,
} INDIA.

\begin{abstract}
In this paper, we introduce the concept of $I_{2}^{*}$-convergence which is closely related to $I_{2}$ - convergence and the concepts $I_{2}$ and $I_{2}^{*}$ - Cauchy double sequences in $p$-adic linear 2-normed space. Also we investigate the relation between these concepts in $p$-adic linear 2-normed spaces.
\end{abstract}

Keywords: 2-normed space, p-adic linear 2-normed space, $I_{2}^{*}$ - convergence, $I_{2}$ - Cauchy double sequence, $I_{2}^{*}-$ Cauchy double sequence.

\section{Introduction}

The idea of $I$ - convergence is based on the notion of the ideal $I$ of subsets of $N$, the set of natural numbers. The notion of ideal convergence for single sequences was introduced first by P.Kostyrko et al $[11,12]$ as an interesting generalization of statistical convergence. F.Nuray and Ruckle [14] independently introduced the same concept as the name generalized statistical convergence.

The concept of a double sequence was initially introduced by Pringsheim [16] in the 1900s and this concept has been studied by many others. A double sequence of real (or complex) numbers is a function from $N \times N$ to $\mathrm{F}$ (where $\mathrm{F}=\mathrm{R}$ or $\mathrm{C}$ ) and we denote a double sequence as $\left(x_{i j}\right)$ where the two subscripts run through the sequence of Natural numbers independent of each other. P.Das et al [4] introduced the concept of $I$ - convergence of double sequences in a metric space and studied some properties. Also, Pratulananda Das and Prasanta Malik [15] defined the concept of $I$ - limit points, $I$ - cluster points, $I$ - limit superior and $I$ - limit inferior of double sequences. Balakrishna Tripathy, Binod Chandra Tripathy [3] introduced the notion of $I$ - convergence and $I$ - Cauchy double sequences and many others discussed the properties of $I$ - convergence, $I^{*}$ - convergence for double sequences and $I$ - Cauchy double sequences (see ([5], [6], [25], for more details).

The concept of linear 2-normed spaces has been investigated by Gähler in 1965 [7] and has been developed extensively in different subjects by others $[9,10,17]$. A.Sahiner et al [20] introduced $I-$ cluster points of convergent sequences in 2-normed linear spaces and Gurdal [8] investigated the relation between $I$ - cluster points and ordinary limit points of sequences in 2-normed spaces. The concept of $I$ - convergence for the double sequences in 2-normed spaces introduced by Saeed Sarabadan and Sorayya Talebi [19]. Saeed Sarabadan, Fatemeh Amoee Arani and Siamak Khalehoghli [18] obtained Condition for the equivalence of I and I*-convergence for double sequences in 2-normed spaces.

Mehmet Acikgoz [13] introduced a very understandable and readable connection between the concepts in $p$-adic numbers, $p$-adic analysis and linear 2-normed spaces. B.Surender Reddy [21] introduced some properties of $p$-adic linear 2-normed spaces and obtained necessary and sufficient conditions for $p$-adic 2-norms to be equivalent on $p$-adic linear 2-normed spaces. Recently B.Surender Reddy and D.Shankaraiah [22, 23, 24] introduced $I$ - convergence, $I^{*}$ - convergence of sequences, $I$ - Cauchy, $I^{*}$ - Cauchy sequences and their properties in $p$-adic linear 2-normed spaces and also introduced ideal convergence of double sequences in $p$-adic linear 2-normed spaces.

The main aim of this paper is we introduce the concept of $I_{2}^{*}$ - convergence of double sequences which is closely related to $I_{2}$ - convergence of double sequences and the concepts $I_{2}$-Cauchy double sequence and $I_{2}^{*}$ - Cauchy double sequence in $p$-adic linear 2-normed space $\left(X, N(\bullet, \bullet)_{p}\right)$. Also we investigate the relation between these concepts in $p$-adic linear 2-normed spaces. 


\section{Preliminaries}

In this paper, we will use the notations; $p$ for a prime number, $\mathrm{Z}$ - the ring of rational integers, $\mathrm{Z}^{+}$- the positive integers, $Q$ - the field of rational numbers, $R$ - the field of real numbers, $R^{+}$- the positive real numbers, $Z_{p}$ - the ring of $p$-adic rational integers, $Q_{p}$ - the field of $p$-adic rational numbers, $C$ - the field of complex numbers and $C_{p}$ - the $p$-adic completion of the algebraic closure of $Q_{p}$.

Definition 2.1: A double sequence $x=\left(x_{i j}\right)$ is said to be convergent to a number $\xi$ in the Pringsheim's sense if for each $\varepsilon>0$ there exists a positive integer m such that $\left|x_{i j}-\xi\right|<\varepsilon$ whenever $i, j \geq m$.Then the number $\xi$ is called the Pringsheim limit of the sequence $\mathrm{x}$ and we write as $P-\lim _{i, j \rightarrow \infty} x_{i j}=\xi$.

Definition 2.2: A double sequence $x=\left(x_{i j}\right)$ is said to be Cauchy sequence if for each $\varepsilon>0$ there exists a positive integer $n_{0}$ such that $\left|x_{i j}-x_{m n}\right|<\varepsilon$ for every $i \geq m \geq n_{0}$ and $j \geq n \geq n_{0}$.

Definition 2.3: A double sequence $x=\left(x_{i j}\right)$ is said to be bounded if there exists a real number $M>0$ such that $\left|x_{i j}\right|<M$ for each $i$ and $j$.

Definition 2.4: Let $K \subset N \times N$ and $K(m, n)=\{(i, j):(i, j) \in K ; i \leq m, j \leq n\}$. If the sequence $\left\{\frac{K(m, n)}{m n}\right\}$ has a limit in Pringsheim's sense then we say that $\mathrm{K}$ has a double natural density and it is denoted as $\lim _{m, n \rightarrow \infty} \frac{K(m, n)}{m n}=\delta_{2}(K)$.

Definition 2.5: A double sequence $x=\left(x_{i j}\right)$ is said to be statistically convergent to a number $\xi$ if for each $\varepsilon>0$, the set $A(\varepsilon)=\left\{(i, j) \in N \times N:\left|x_{i j}-\xi\right| \geq \varepsilon\right\}$ has double natural density zero. If $x=\left(x_{i j}\right)$ is statistically convergent to $\xi$ then we write $S t-\lim _{i, j \rightarrow \infty} x_{i j}=\xi$.

Definition 2.6: Let $I_{2}$ be an ideal in $N \times N$. A double sequence $x=\left(x_{i j}\right)$ is said to be $I_{2}-$ convergent to $\mathrm{L}$ in Pringsheim's sense if for each $\varepsilon>0$, the set $\left\{(i, j) \in N \times N:\left|x_{i j}-L\right| \geq \varepsilon\right\} \in I_{2}$ and L is called $I_{2}-$ limit of $x=\left(x_{i j}\right)$ and we write $I_{2}-\lim _{i, j \rightarrow \infty} x_{i j}=L$.

Definition 2.7: A double sequence $x=\left(x_{i j}\right)$ is said to be $I_{2}^{*}$-convergent to $\xi$ if there exists a set $M=\{(i, j): i, j=1,2,3, \ldots \ldots ..\} \in F\left(I_{2}\right)\left(i . e,(N \times N)-M \in I_{2}\right)$ such that $\lim _{i, j \rightarrow \infty} x_{i j}=\xi$ and $\xi$ is called $I_{2}^{*}-\operatorname{limit}$ of $x=\left(x_{i j}\right)$ and we write $I_{2}^{*}-\lim x_{i j}=\xi$.

Definition 2.8: A double sequence $x=\left(x_{i j}\right)$ is $I_{2}$ - convergent to zero in Pringsheim's sense is called $I_{2}-$ null double sequence in Pringsheim's sense.

Definition 2.9: Let $X$ be a linear space of dimension greater than 1 over $K$, where $K$ is the real or complex numbers field. Suppose $N(\bullet, \bullet)$ be a non-negative real valued function on $X \times X$ satisfying the following conditions:

$\left(2-N_{1}\right): N(x, y)>0$ and $N(x, y)=0$ if and only if $x$ and $y$ are linearly dependent vectors, 
$\left(2-N_{2}\right): N(x, y)=N(y, x)$ for all $x, y \in X$,

$\left(2-N_{3}\right): N(\lambda x, y)=|\lambda| N(x, y)$ for all $\lambda \in K$ and $x, y \in X$,

$\left(2-N_{4}\right): N(x+y, z) \leq N(x, z)+N(y, z)$ for all $x, y, z \in X$.

Then $N(\bullet, \bullet)$ is called a 2-norm on $X$ and the pair $(X, N(\bullet, \bullet))$ is called a linear 2-normed space.

Definition 2.10: Suppose a mapping $d_{p}: X \times X \times X \rightarrow R$ on a non-empty set $X$ satisfying the following conditions, for all $x, y, z \in X$

$\left.D_{1}\right)$ For any two different elements $\mathrm{x}$ and $\mathrm{y}$ in $\mathrm{X}$ there is an element $z$ in $X$ such that $d_{p}(x, y, z) \neq 0$.

$\left.D_{2}\right) d_{p}(x, y, z)=0$ when two of three elements are equal.

$\left.D_{3}\right) d_{p}(x, y, z)=d_{p}(x, z, y)=d_{p}(y, z, x)$.

$\left.D_{4}\right) d_{p}(x, y, z) \leq d_{p}(x, y, w)+d_{p}(x, w, z)+d_{p}(w, y, z)$ for any $w$ in $X$. Then $d_{p}$ is called $p$-adic

2-metric on $X$ and the pair $\left(\mathrm{X}, d_{p}\right)$ is called $p$-adic 2-metric space. If $p$-adic 2-metric also satisfies the condition $d_{p}(x, y, z) \leq \max \left\{d_{p}(x, y, w), d_{p}(x, w, z), d_{p}(y, w, z)\right\}$ for $x, y, z, w \in X$, then $d_{p}$ is called a $p$-adic ultra 2-metric and the pair $\left(X, d_{p}\right)$ is called a $p$-adic ultra 2-metric space.

Definition 2.11: Let $X$ be a linear space of dimension greater than 1 over $K$, where $K$ is the real or complex numbers field. Suppose $N(\bullet, \bullet)_{p}$ be a non-negative real valued function on $X \times X$ satisfying the following conditions:

$\left(2-p N_{1}\right): N(x, z)_{p}=0$ if and only if $x$ and $z$ are linearly dependent vectors.

$\left(2-p N_{2}\right): N(x y, z)_{p}=N(x, z)_{p} \cdot N(y, z)_{p}$ for all $x, y, z \in X$,

$\left(2-p N_{3}\right): N(x+y, z)_{p} \leq N(x, z)_{p}+N(y, z)_{p}$ for all $x, y, z \in X$,

$\left(2-p N_{4}\right): N(\lambda x, z)_{p}=|\lambda| N(x, z)_{p}$ for all $\lambda \in K$ and $x, z \in X$.

Then $N(\bullet, \bullet)_{p}$ is called a $p$-adic 2-norm on $X$ and the pair $\left(X, N(\bullet, \bullet)_{p}\right)$ is called $p$-adic linear 2-normed space.

For every $p$-adic linear 2-normed space $\left(X, N(\bullet, \bullet)_{p}\right)$ the function defined on $X \times X \times X$ by $d_{p}(x, y, z)=N(x-z, y-z)_{p} \quad$ is a $p$-adic 2-metric. Thus every $p$-adic linear 2-normed space $\left(X, N(\bullet, \bullet)_{p}\right)$ will be considered to be a $p$-adic 2-metric space with this 2-metric. A double sequence $\left(x_{i j}\right)$ of $p$-adic 2-metric space $\left(X, d_{p}\right)$ converges to $x \in X$ in $p$-adic 2-metric if for every $\varepsilon>0$, there is an $l \geq 1$ such that $d_{p}\left(x_{i j}, x, z\right)=N\left(x_{i j}-z, x-z\right)_{p}<\varepsilon$ for every $i, j \geq l$. For the given two double sequences of $p$-adic 2-metric space $\left(X, d_{p}\right)$ which are $\left(x_{i j}\right)$ and $\left(y_{i j}\right)$ converges to $x, y \in X$ in the $p$-adic 2-metric space respectively, then the double sequence of sums $x_{i j}+y_{i j}$ and the product $x_{i j} y_{i j}$ converges to the sum $x+y$ and to the product $x y$ of the limits of initial double sequences.

A double sequence $\left(x_{i j}\right)$ of $p$-adic 2-metric space $\left(X, d_{p}\right)$ is a Cauchy double sequence with respect to the $p$ adic 2-metric if for each $\varepsilon>0$, there is an $l \geq 1$ such that $d_{p}\left(x_{i j}, x_{m n}, z\right)=N\left(x_{i j}-z, x_{m n}-z\right)_{p}<\varepsilon$, for every $i \geq m \geq l, j \geq n \geq l$.

Definition 2.12: A double sequence $x=\left(x_{i j}\right)$ in a $p$-adic linear 2-normed space $\left(X, N(\bullet, \bullet)_{p}\right)$ is said to be convergent to $l \in X$ if for each $\varepsilon>0$ there exists $m \in N$ such that $N\left(x_{i j}-l, z\right)_{p}<\varepsilon$ for each 
$i, j \geq m$ and for each $z \in X$. If $x=\left(x_{i j}\right)$ is convergent to $l$ then we write $\lim _{i, j \rightarrow \infty} x_{i j}=l$ or $x_{i j} \stackrel{\left[N(\bullet, \bullet)_{p}\right]_{X}}{\longrightarrow} l$.

Definition 2.13: A double sequence $x=\left(x_{i j}\right)$ in a $p$-adic linear 2-normed space $\left(X, N(\bullet, \bullet)_{p}\right)$ is said to be bounded if for each non zero $z \in X$ and for all $i, j \in N$ there exists $M>0$ such that $N\left(x_{i j}, z\right)_{p}<M$. Note that a convergent double sequence need not be bounded.

Definition 2.14: A double sequence $x=\left(x_{i j}\right)$ in a $p$-adic linear 2-normed space $\left(X, N(\bullet, \bullet)_{p}\right)$ is said to be Cauchy double sequence if for each $\varepsilon>0$ there exists a positive integer $n_{0}$ such that $N\left(x_{i j}-x_{m n}, z\right)_{p}<\varepsilon$ for every $i \geq m \geq n_{0}$ and $j \geq n \geq n_{0}$.

A $p$-adic linear 2-normed space $\left(X, N(\bullet, \bullet)_{p}\right)$ is called complete if every Cauchy sequence is convergent in $p$-adic linear 2-normed space. A p-adic linear 2-normed space $\left(X, N(\bullet, \bullet)_{p}\right)$ is called $p$-adic 2Banach space if $p$-adic linear 2-normed space is complete.

Proposition 2.15: If a double sequence $\left\{x_{i j}\right\}$ in a $p$-adic linear 2-normed space $\left(X, N(\bullet, \bullet)_{p}\right)$ is convergent to $x \in X$, then $\lim _{i, j \rightarrow \infty} N\left(x_{i j}, z\right)_{p}=N(x, z)_{p}$ for each $\mathrm{z} \in \mathrm{X}$.

Proposition 2.16: If $\lim _{i, j \rightarrow \infty} N\left(x_{i j}, z\right)_{p}$ exists then we say that $\left(x_{i j}\right)$ is a Cauchy sequence with respect to $N(\bullet, \bullet)_{p}$.

Proof: Let us suppose that $\lim _{i, j \rightarrow \infty} N\left(x_{i j}, z\right)_{p}=x$. Then we can obtain a constant $M_{1}$ such that $i, j>M_{1}$ $\Rightarrow N\left(x-x_{i j}, z\right)_{p}<\frac{\varepsilon}{2}$. If $i, j, m, n>M_{1}$ then $N\left(x-x_{i j}, z\right)_{p}<\frac{\varepsilon}{2}$ and $N\left(x-x_{m n}, z\right)_{p}<\frac{\varepsilon}{2}$, hence by using the triangle inequality, we have $N\left(x_{i j}-x_{m n}, z\right)_{p}=N\left(x_{i j}-x+x-x_{m n}, z\right)_{p}$ $\leq N\left(x_{i j}-x, z\right)_{p}+N\left(x-x_{m n}, z\right)_{p}<\frac{\varepsilon}{2}+\frac{\varepsilon}{2}=\varepsilon$.

$\Rightarrow\left(x_{i j}\right)$ is a Cauchy sequence with respect to $N(\bullet, \bullet)_{p}$.

\section{Main Results}

In this section, we introduce the concept of $I_{2}^{*}$ - convergence of double sequences which is closely related to $I_{2}$ - convergence of double sequences in p-adic linear 2-normed space $\left(X, N(\bullet \bullet \bullet)_{p}\right)$ and we introduce the concepts $I_{2}$ - Cauchy double sequence and $I_{2}^{*}$ - Cauchy double sequence in $p$-adic linear 2normed space $\left(X, N(\bullet, \bullet)_{p}\right)$. Also we investigate the relation between these concepts in $p$-adic linear 2 normed spaces.

A family of sets $I \subseteq 2^{Y}$ (power sets of $Y$ ) is said to be an ideal if $\Phi \in I, I$ is additive i.e., $A, B \in I \Rightarrow A \cup B \in I$ and hereditary i.e., $A \in I, B \subseteq A \Rightarrow B \in I$.

A non empty family of sets $F \subset 2^{Y}$ is a filter on $Y$ if and only if $\Phi \notin F, A \cap B \in F$ for each $A, B \in F$, and any subset of an element of $F$ is in $F$. An ideal $I$ is called non-trivial if $I \neq \Phi$ and $Y \notin I$. Clearly $I$ is a non-trivial ideal if and only if $F=F(I)=\{Y-A: A \in I\}$ is a filter in 
$Y$, called the filter associated with the ideal $I$. A non-trivial ideal $I$ is called admissible if and only if $\{\{n\}: n \in Y\} \subset I$.

An admissible ideal $I \subset 2^{Y}$ is said to have the property (AP) if for any sequence $\left\{A_{1}, A_{2}, A_{3}, \ldots\right\}$ of mutually disjoint sets of $I$ there is a sequence $\left\{B_{1}, B_{2}, B_{3}, \ldots\right\}$ of sets such that each symmetric difference $A_{i} \Delta B_{i}, i=1,2,3 \ldots .$, is finite and $B=\cup_{i=1}^{\infty} B_{i} \in I$.

In order to distinguish between the ideals of $N$ and $N \times N$ we shall denote the ideals of $\mathrm{N}$ by I and ideals of $N \times N$ by $I_{2}$. In general, there is no connection between $I$ and $I_{2}$.

A non trivial ideal $I_{2}$ in $N \times N$ is called strongly admissible if $\{i\} \times N$ and $N \times\{i\}$ belong to $I_{2}$ for each $i \in N$. It is clear that a strongly admissible ideal is admissible also.

Let $I_{0}=\left\{A \subset N \times N:(\exists m(A) \in N)(i, j \geq m(A) \Rightarrow(i, j) \in(N \times N)-A\}\right.$. Then $I_{0}$ is a non trivial strongly admissible ideal and $I_{2}$ is strongly admissible if and only if $I_{0} \subseteq I_{2} . I_{2} \subset 2^{N \times N}$ is a non trivial ideal if and only if the class $F=F(I)=\left\{(N \times N)-A: A \in I_{2}\right\}$ is a filter in $N \times N$.

An admissible ideal $I_{2} \subset 2^{N \times N}$ satisfies the condition $\left(A P_{2}\right)$ if for each countable family of disjoint sets $\left\{A_{1}, A_{2}, A_{3}, \ldots\right\}$ belongs to $I_{2}$, there exists a countable family of sets $\left\{B_{1}, B_{2}, B_{3}, \ldots\right\}$ such that $A_{j} \Delta B_{j}$ is included in the finite union of rows and columns in $N \times N$ for each $j \in N$ and $B=\bigcup_{j=1}^{\infty} B_{j} \in I_{2}$ ( hence $B_{j} \in I_{2}$ for each $j \in N$ ).

Definition 3.1: A double sequence $x=\left(x_{i j}\right)$ in a $p$-adic linear 2-normed space $\left(X, N(\bullet, \bullet)_{p}\right)$ is said to be $I_{2}$ - convergent to $l \in X \quad$ if for each $\varepsilon>0$ and non zero $z \in X$, the set $A(\varepsilon)=\left\{(i, j) \in N \times N: N\left(x_{i j}-l, z\right)_{p} \geq \varepsilon\right\} \in I_{2} \quad$ and $\quad l \quad$ is called the $I_{2}$-limit of the sequence $x=\left(x_{i j}\right)$.

If $x=\left(x_{i j}\right)$ is $I_{2}-$ convergent to $l$, then we write $I_{2}-\lim _{i, j \rightarrow \infty} x_{i j}=l$ or $I_{2}-\lim _{i, j \rightarrow \infty} N\left(x_{i j}-l, z\right)_{p}=0$ or $I_{2}-\lim _{i, j \rightarrow \infty} N\left(x_{i j}, z\right)_{p}=N(l, z)_{p}$ for each non zero $z \in X$.

Now introducing the definition of $I_{2}^{*}$ - convergence for double sequence $x=\left(x_{i j}\right)$ which is closely related to $I_{2}$ - convergence of double sequence $x=\left(x_{i j}\right)$ in a $p$-adic linear 2-normed space as follows.

Definition 3.2: A double sequence $x=\left(x_{i j}\right)$ in a $p$-adic linear 2-normed space $\left(X, N(\bullet, \bullet)_{p}\right)$ is said to be $I_{2}^{*}$ - convergent to $l \in X$ if there exists a set $M \in F\left(I_{2}\right)$ (i.e., $(N \times N)-M \in I_{2}$ ) such that $\lim _{i, j \rightarrow \infty} x_{i j}=l$ for $(i, j) \in M$ and $l$ is called the $I_{2}^{*}-$ limit of the sequence $x=\left(x_{i j}\right)$.

If $x=\left(x_{i j}\right)$ is $I_{2}^{*}-$ convergent to $l$, then we write $I_{2}^{*}-\lim _{i, j \rightarrow \infty} x_{i j}=l$ or $I_{2}^{*}-\lim _{i, j \rightarrow \infty} N\left(x_{i j}-l, z\right)_{p}=0$ or $I_{2}^{*}-\lim _{i, j \rightarrow \infty} N\left(x_{i j}, z\right)_{p}=N(l, z)_{p}$ for each non zero $z \in X$. 
Lemma 3.3: Let $\left\{P_{i}\right\}_{i=1}^{\infty}$ be a countable collection of subsets of $N \times N$ such that $\left\{P_{i}\right\}_{i=1}^{\infty} \in F\left(I_{2}\right)$ for each $i$, where $F\left(I_{2}\right)$ is a filter associated with a strongly admissible ideal $I_{2}$ with property $\left(\mathrm{AP}_{2}\right)$. Then there is a set $P \subset N \times N$ such that $P \in F\left(I_{2}\right)$ and the set $P-P_{i}$ is finite for all $i$.

Lemma 3.4: Let $I_{2} \subset 2^{N \times N}$ be a strongly admissible ideal and $\left(X, N(\bullet, \bullet)_{p}\right)$ be a $p$-adic linear 2normed space. If $I_{2}^{*}-\lim _{i, j \rightarrow \infty} x_{i j}=l$, then $I_{2}-\lim _{i, j \rightarrow \infty} x_{i j}=l$ for each non zero $z \in X$.

Proof: Suppose $I_{2}^{*}-\lim _{i, j \rightarrow \infty} x_{i j}=l$. Then there exists $H \in I_{2}$ such that for $M=(N \times N)-H \in F\left(I_{2}\right)$ we have

$\lim _{i, j \rightarrow \infty} x_{i j}=l,(i, j) \in M$, for each non zero $z \in X$

Let $\varepsilon>0$. By virtue of equation (3.5) there exists a positive integer $n_{1}$ such that $N\left(x_{i j}-l, z\right)_{p}<\varepsilon$ for every $(i, j) \in M$ with $i, j \geq n_{1}$.

Let $A=\left\{1,2,3, \ldots \ldots, n_{1}-1\right\}, B=\left\{(i, j) \in M: N\left(x_{i j}-l, z\right)_{p} \geq \varepsilon\right\}$.Then $\quad$ it $\quad$ is $\quad$ clear $\quad$ that $B \subset(A \times N) \cup(N \times A)$ and therefore $B \in I_{2}$. Obviously the set

$\left\{(i, j) \in N \times N: N\left(x_{i j}-l, z\right)_{p} \geq \varepsilon\right\} \subset B \cup H$ and therefore the set

$\left\{(i, j) \in N \times N: N\left(x_{i j}-l, z\right)_{p} \geq \varepsilon\right\} \in I_{2}$ for each non zero $z \in X$. This implies that $I_{2}-\lim _{i, j \rightarrow \infty} x_{i j}=l$, for each non zero $z \in X$.

Theorem 3.6: Let $I_{2} \subset 2^{N \times N}$ be a strongly admissible ideal with property $\left(A P_{2}\right)$ and $\left(X, N(\bullet, \bullet)_{p}\right)$ be a $p$-adic linear 2-normed space. Then for an arbitrary double sequence $x=\left(x_{i j}\right)$ of elements of $X$, if $I_{2}-\lim _{i, j \rightarrow \infty} x_{i j}=l$ then $I_{2}^{*}-\lim _{i, j \rightarrow \infty} x_{i j}=l$.

Proof: Suppose that $I_{2}-\lim _{i, j \rightarrow \infty} x_{i j}=l$. Then for any $\varepsilon>0$ and non zero $z \in X$, $A(\varepsilon)=\left\{(i, j) \in N \times N: N\left(x_{i j}-l, z\right)_{p} \geq \varepsilon\right\} \in I_{2}$.

Now put $A_{1}=\left\{(i, j) \in N \times N: N\left(x_{i j}-l, z\right)_{p} \geq 1\right\}$ and $A_{k}=\left\{(i, j) \in N \times N: \frac{1}{k} \leq N\left(x_{i j}-l, z\right)_{p}<\frac{1}{k-1}\right\}$ for $k \geq 2$ and for each non zero $z \in X$. It is clear that $A_{m} \cap A_{n}=\phi$ for $m \neq n$ and $A_{m} \in I_{2}$ for each $m \in N$. By virtue of $\left(A P_{2}\right)$ there exists a countable family of sets $\left\{B_{1}, B_{2}, B_{3}, \ldots\right\}$ such that $A_{m} \Delta B_{m}$ is a included in finite union of rows and columns in $N \times N$ for each $m \in N$ and $B=\bigcup_{m=1}^{\infty} B_{m} \in I_{2}$. Put $M=(N \times N)-B$ and to prove the theorem it is sufficient to prove that $\lim _{i, j \rightarrow \infty} x_{i j}=l$ for $(i, j) \in M$.

Let $\delta>0$. Choose $k \in N$ such that $\frac{1}{k}<\delta$. Then we have

$\left\{(i, j) \in N \times N: N\left(x_{i j}-l, z\right)_{p} \geq \delta\right\} \subseteq \bigcup_{m=1}^{k} A_{m}$

Since $A_{m} \Delta B_{m}, \quad m=1,2,3, \ldots . ., k \quad$ is $\quad$ a finite $\quad$ set, there exists $n_{0} \in N \quad$ such that $\left(\bigcup_{m=1}^{k} B_{m}\right) \cap\left\{(i, j): i, j \geq n_{0}\right\}=\left(\bigcup_{m=1}^{k} A_{m}\right) \cap\left\{(i, j): i, j \geq n_{0}\right\} . \quad$ If $\quad i, j>n_{0} \quad$ and $\quad(i, j) \in M$, 
then $(i, j) \notin B$. This implies that $(i, j) \notin \bigcup_{m=1}^{k} B_{m}$ and therefore $(i, j) \notin \bigcup_{m=1}^{k} A_{m}$. Hence for every $i, j>n_{0}$ and $(i, j) \in M$, we have by equation (3.7), $N\left(x_{i j}-l, z\right)_{p}<\delta$, for each $z \in X$.

$\Rightarrow \lim _{i, j \rightarrow \infty} x_{i j}=l$, for $(i, j) \in M$.

Thus $I_{2}^{*}-\lim _{i, j \rightarrow \infty} x_{i j}=l$.

From the Lemma (3.4) and Theorem (3.6) we obtain the following Lemma which gives the equivalence between $I_{2}$-convergence and $I_{2}^{*}$ - convergence in $p$-adic linear 2-normed spaces.

Lemma 3.8: Let $I_{2} \subset 2^{N \times N}$ be a strongly admissible ideal with the property $\left(A P_{2}\right)$ and $\left(X, N(\bullet, \bullet)_{p}\right)$ be a $p$-adic linear 2-normed space. Then a double sequence in $X$ is $I_{2}$-convergent to $l$ in $X$ if and only if it is $I_{2}^{*}$ - convergent to $l$ in $X$.

Definition 3.9: Let $\left(X, N(\bullet, \bullet)_{p}\right)$ be a $p$-adic linear 2-normed space and $x \in X$. Then $x$ is called accumulation point of $X$ if there exists a sequence $\left(x_{k}\right)$ of distinct elements of $X$ such that $x_{k} \neq x$ for any $k$ and $x_{n} \stackrel{N(\bullet, \bullet)_{p}}{\longrightarrow} x$.

Theorem 3.10: Let $I_{2} \subset 2^{N \times N}$ be a strongly admissible ideal and $\left(X, N(\bullet, \bullet)_{p}\right)$ be a $p$-adic linear 2normed space. If $X$ has at least one accumulation point for any arbitrary double sequence $x=\left(x_{i j}\right)$ in $X$ and for each $a \in X, I_{2}-\lim _{i, j \rightarrow \infty} x_{i j}=a$ implies $I_{2}^{*}-\lim _{i, j \rightarrow \infty} x_{i j}=a$, then $I_{2}$ has the property $\left(A P_{2}\right)$.

Proof: Suppose that $a \in X$ is accumulation point of $X$. Then there is a sequence $\left(b_{k}\right)$ of distinct elements of $X$ such that $b_{k} \neq a$ for any $k$, and $b_{k} \stackrel{N(\bullet, \bullet)_{p}}{\longrightarrow} a$.

Put $\varepsilon_{k}^{(z)}=N\left(b_{k}-a, z\right)_{p}$ for $k \in N$. Let $\left(A_{j}\right)_{j \in N}$ be a disjoint family of non empty sets from $I_{2}$. Define a sequence $\left(x_{m n}\right)$ for all $z \in X$ such that

(i) . $x_{m n}=b_{j}$ if $(m, n) \in A_{j}$ and

(ii) . $x_{m n}=a$ if $(m, n) \notin A_{j}$ for any $j$.

Let $\delta>0$ be given and $z_{0} \in X$. Choose $k \in N$ such that $\varepsilon_{k}^{\left(z_{0}\right)}<\delta$. Then we have

$A^{z_{0}}(\delta)=\left\{(m, n): N\left(x_{m n}-a, z_{0}\right)_{p} \geq \delta\right\} \subseteq A_{1} \bigcup A_{2} \cup \ldots \ldots . . \cup A_{k}$. Hence $\quad A^{z_{0}}(\delta) \in I_{2} \quad$ and $\quad$ so $I_{2}-\lim _{m, n \rightarrow \infty} x_{m n}=a$.

By virtue of our assumption, we have $I_{2}^{*}-\lim _{m, n \rightarrow \infty} x_{m n}=a$. Hence there exists a set $B \in I_{2}$ such that $M=(N \times N)-B \in F\left(I_{2}\right)$ and $\lim _{m, n \rightarrow \infty} x_{m n}=a,(m, n) \in M$

Let $B_{j}=A_{j} \cap B$ for $j \in N$. Then $B_{j} \in I_{2}$ for each $j \in N$ and $\bigcup_{j=1}^{\infty} B_{j}=B \cap\left(\bigcup_{j=1}^{\infty} A_{j}\right) \subseteq B$ and so $\bigcup_{j=1}^{\infty} B_{j} \in I_{2}$, fix $j \in N$ 
If $A_{j} \cap M$ is not included in the finite union of rows and columns in $N \times N$, then $M$ must contain an infinite sequence of elements $\left(m_{k}, n_{k}\right)$ and $\lim _{m_{k}, n_{k} \rightarrow \infty} x_{m_{k} n_{k}}=b_{j} \neq a$ for all $k \in N$, which contradicts equation (3.11). Hence $A_{j} \cap M$ must be contained in the finite union of rows and columns in $N \times N$. Thus

$$
\begin{aligned}
A_{j} \Delta B_{j} & =\left(A_{j}-B_{j}\right) \cup\left(B_{j}-A_{j}\right) \\
& =A_{j}-B_{j} \text { since } B_{j}-A_{j}=\phi \\
& =A_{j}-\left(A_{j} \cap B\right) \\
& =A_{j}-B=A_{j} \cap M \text { is also included in the finite union of rows and columns. Thus the ideal } I_{2} \text { has }
\end{aligned}
$$
the property $\left(A P_{2}\right)$.

Definition 3.12: Let $I_{2} \subset 2^{N \times N}$ be a strongly admissible ideal and $\left(X, N(\bullet, \bullet)_{p}\right)$ be a $p$-adic linear 2normed space. A double sequence $x=\left(x_{i j}\right)$ in $X$ is said to be $I_{2}$ - Cauchy double sequence in $X$, if for each $\varepsilon>0$ and non zero $z \in X$, there exists $m=m(\varepsilon, z), n=n(\varepsilon, z) \in N \quad$ such that $\left\{(i, j) \in N \times N: N\left(x_{i j}-x_{m n}, z\right)_{p} \geq \varepsilon\right\} \in I_{2}$.

Definition 3.13: Let $I_{2} \subset 2^{N \times N}$ be a strongly admissible ideal and $\left(X, N(\bullet, \bullet)_{p}\right)$ be a $p$-adic linear 2normed space. A double sequence $x=\left(x_{i j}\right)$ in $X$ is said to be $I_{2}^{*}$ - Cauchy double sequence in $X$, if for each $\varepsilon>0$ and non zero $z \in X$, there exists a set $M \in F\left(I_{2}\right)\left(\right.$ i.e., $\left.\mathrm{H}=(N \times N)-M \in I_{2}\right)$ such that the double sequence $\left(x_{m n}\right)_{(m, n) \in M}$ is a Cauchy sequence in $X$. i.e., $\lim _{i, j, m, n \rightarrow \infty} N\left(x_{i j}-x_{m n}, z\right)_{p}=0$ for each non zero $z \in X$ and $(i, j),(m, n) \in M$.

Theorem 3.14: Let $I_{2} \subset 2^{N \times N}$ be a strongly admissible ideal and $\left(X, N(\bullet, \bullet)_{p}\right)$ be a $p$-adic linear 2normed space. If a double sequence $x=\left(x_{i j}\right)$ is $I_{2}^{*}$ - Cauchy double sequence in $X$, then it is $I_{2}$-Cauchy double sequence in $X$.

Proof: Suppose $x=\left(x_{i j}\right)$ is $I_{2}^{*}$-Cauchy double sequence a in $p$-adic linear 2-normed space $\left(X, N(\bullet, \bullet)_{p}\right)$. Then there exists a set $M \in F\left(I_{2}\right)\left(i . e ., \mathrm{H}=(N \times N)-M \in I_{2}\right)$ such that $N\left(x_{i j}-x_{m n}, z\right)_{p}<\varepsilon$ for every $\varepsilon>0$ and for all $(\mathrm{i}, \mathrm{j}),(\mathrm{m}, \mathrm{n}) \in M ; \mathrm{i}, \mathrm{j}, \mathrm{m}, \mathrm{n} \geq l$ and $l=l(\varepsilon) \in \mathrm{N}$.

Now $A(\varepsilon)=\left\{(i, j) \in N \times N: N\left(x_{i j}-x_{m n}, z\right)_{p} \geq \varepsilon\right\}$

$$
\subset H \bigcup[M \cap((\{1,2,3, \ldots . ., l-1\} \times N) \bigcup(N \times\{1,2,3, \ldots \ldots ., l-1\}))]
$$

Since $I_{2}$ is a strongly admissible ideal, therefore

$$
H \cup[M \cap((\{1,2,3, \ldots \ldots, l-1\} \times N) \bigcup(N \times\{1,2,3, \ldots \ldots ., l-1\}))] \in I_{2} .
$$

From equation (3.15) and by the definition of ideal, $A(\varepsilon) \in \mathrm{I}_{2}$. This shows that the double sequence $x=\left(x_{i j}\right)$ is $I_{2}$ - Cauchy double sequence in $X$.

Now we will prove in the following theorem that $I_{2}^{*}$ - convergence implies $I_{2}$ - Cauchy condition in $p$ - adic linear 2-normed space.

Theorem 3.16: Let $I_{2} \subset 2^{N \times N}$ be a strongly admissible ideal and $\left(X, N(\bullet, \bullet)_{p}\right)$ be a $p$-adic linear 2normed space. If a double sequence $\left(x_{i j}\right)$ in $X$ is $I_{2}^{*}$ - convergent to $x \in X$, then $\left(x_{i j}\right)$ is $I_{2}$ - Cauchy double sequence in $X$. 
Proof: Suppose a double sequence $\left(x_{i j}\right)$ in a $p$-adic linear 2-normed space $\left(X, N(\bullet, \bullet)_{p}\right)$ is $I_{2}^{*}-$ convergent to $x \in X$. Then there exists a set $M \in F\left(I_{2}\right)$

(i.e., $\left.\mathrm{H}=(N \times N)-M \in I_{2}\right)$ such that $\lim _{m, n \rightarrow \infty} x_{m n}=x,(m, n) \in M$. It shows that there exists $k_{0}=k_{0}(\varepsilon)$ such that

$N\left(x_{m n}-x, z\right)_{p}<\frac{\varepsilon}{2}$, for every $\varepsilon>0$, non zero $z \in X$ and $m, n>k_{0}$

For $(i, j),(m, n) \in M$ and $i, j, m, n>k_{0}$,

$$
\begin{aligned}
N\left(x_{i j}-x_{m n}, z\right)_{p} & =N\left(x_{i j}-x+x-x_{m n}, z\right)_{p} \\
& =N\left(\left(x_{i j}-x\right)-\left(x_{m n}-x\right)_{,} z\right)_{p} \\
& \leq N\left(x_{i j}-x, z\right)_{p}+N\left(x_{m n}-x, z\right)_{p} \\
& <\frac{\varepsilon}{2}+\frac{\varepsilon}{2}, \quad \text { by equation (3.17) } \\
& =\varepsilon, \text { for each } \varepsilon>0, \text { non zero } z \in X \text { and } i, j, m, n>k_{0} .
\end{aligned}
$$

Therefore $\lim _{i, j, m, n \rightarrow \infty} N\left(x_{i j}-x_{m n}, z\right)_{p}=0$ which implies $\left(x_{i j}\right)_{(i, j) \in M}$ is a Cauchy double sequence in $X$ and hence $\left(x_{m n}\right)_{(m, n) \in N \times N}$ is a $I_{2}^{*}$ - Cauchy double sequence in $X$. By Theorem $(3.14),\left(x_{m n}\right)_{(m, n) \in N \times N}$ is a $I_{2}$ - Cauchy double sequence in $X$.

From Theorem (3.16) and Lemma (3.8) we have the following corollary which gives the relation between $I_{2}$ - convergence and $I_{2}$ - Cauchy double sequence in a $p$-adic linear 2-normed space $\left(X, N(\bullet, \bullet)_{p}\right)$.

Corollary 3.18: Let $I_{2} \subset 2^{N \times N}$ be a strongly admissible ideal with the property $\left(A P_{2}\right)$ and $\left(X, N(\bullet, \bullet)_{p}\right)$ be a $p$-adic linear 2-normed space. If a double sequence $\left(x_{i j}\right)$ is $I_{2}$-convergent to $x$ in $X$, then $\left(x_{i j}\right)$ is $I_{2}$ - Cauchy double sequence in $X$.

Finally, we will give the following Theorem which states the equivalence of $I_{2}$ - Cauchy double sequence and $I_{2}^{*}$ - Cauchy double sequence in a $p$-adic linear 2-normed space $\left(X, N(\bullet, \bullet)_{p}\right)$ in the case $I_{2}$ has the property $\left(A P_{2}\right)$.

Theorem 3.19: Let $I_{2} \subset 2^{N \times N}$ be a strongly admissible ideal with the property $\left(A P_{2}\right)$ and $\left(X, N(\bullet, \bullet)_{p}\right)$ be a $p$-adic linear 2-normed space. Then a double sequence $x=\left(x_{i j}\right)$ is $I_{2}$-Cauchy double sequence in $X$ if and only if $x=\left(x_{i j}\right)$ is $I_{2}^{*}$ - Cauchy double sequence in $X$.

Proof: Suppose a double sequence $x=\left(x_{i j}\right)$ is $I_{2}^{*}$ - Cauchy double sequence in a $p$-adic linear 2-normed space $\left(X, N(\bullet, \bullet)_{p}\right)$.Then by Theorem (3.14), $x=\left(x_{i j}\right)$ is $I_{2}$ - Cauchy double sequence in $X$.

Now it is sufficient to prove that, if a double sequence $x=\left(x_{i j}\right)$ is $I_{2}$-Cauchy double sequence in $X$, then it is $I_{2}^{*}$ - Cauchy double sequence in $X$.Suppose $x=\left(x_{i j}\right)$ is $I_{2}$ - Cauchy double sequence in $X$. Then there exists $m=m(\varepsilon), n=n(\varepsilon) \in N$ such that $A(\varepsilon)=\left\{(m, n): N\left(x_{i j}-x_{m n}, z\right)_{p} \geq \varepsilon\right\} \in I_{2}$ for each $\varepsilon>0$ and non zero $z \in X$. 
Let $P_{k}=\left\{(i, j) \in N \times N: N\left(x_{i j}-x_{m_{k} n_{k}}, z\right)_{p}<\frac{1}{k}\right\}, k \in N$ where $m_{k}=m\left(\frac{1}{k}\right), n_{k}=n\left(\frac{1}{k}\right)$.

Since $H_{k}=(N \times N)-P_{k}=\left\{(i, j) \in N \times N: N\left(x_{i j}-x_{m_{k} n_{k}}, z\right)_{p} \geq \frac{1}{k}\right\} \in I_{2}$ for each $k \in N$ and non zero $z \in X$, therefore $P_{k} \in F\left(I_{2}\right)$, for each $k \in N$.

Since $I_{2}$ has the property $\left(A P_{2}\right)$, then by Lemma (3.3) there exists a set $P \subset N \times N$ such that $P \in F\left(I_{2}\right)$ and $P-P_{k}$ is finite for all $k \in N$. Now we have to show that $\lim _{i, j, m, n \rightarrow \infty} N\left(x_{i j}-x_{m n}, z\right)_{p}=0$, for $(i, j),(m, n) \in P$ and for each non zero $z \in X$. For this, let $\varepsilon>0$ and $l \in N$ be such that $l>\frac{2}{\varepsilon}$. If $(i, j),(m, n) \in P$, then $P-P_{l}$ is finite, so there exists $\alpha=\alpha(l)$ such that $(i, j),(m, n) \in P_{l}$ for all $i, j, m, n>\alpha(l)$. Therefore

$N\left(x_{i j}-x_{m_{l} n_{l}}, z\right)_{p}<\frac{1}{l}$ and $N\left(x_{m n}-x_{m_{l} n_{l}}, z\right)_{p}<\frac{1}{l}$ for all $i, j, m, n>\alpha(l)$ and non zero $z \in X$.

Now $N\left(x_{i j}-x_{m n}, z\right)_{p}=N\left(x_{i j}-x_{m_{l} n_{l}}+x_{m_{l} n_{l}}-x_{m n}, z\right)_{p}$

$$
\begin{aligned}
& =N\left(\left(x_{i j}-x_{m_{l} n_{l}}\right)-\left(x_{m n}-x_{m_{l} n_{l}}\right), z\right)_{p} \\
& \leq N\left(x_{i j}-x_{m_{l} n_{l}}, z\right)_{p}+N\left(x_{m n}-x_{m_{l} n_{l}}, z\right)_{p} \\
& <\frac{1}{l}+\frac{1}{l}=\frac{2}{l}<\varepsilon \text {, for all } i, j, m, n>\alpha(l) \text { and non zero } z \in X .
\end{aligned}
$$

Hence for each $\varepsilon>0$ there exists $\alpha=\alpha(\varepsilon)$ such that for $i, j, m, n>\alpha(\varepsilon)$ and $(i, j),(m, n) \in P \in F\left(I_{2}\right)$, we have $N\left(x_{i j}-x_{m n}, z\right)_{p}<\varepsilon$ for each non zero $z \in X$. This shows that the sequence $x=\left(x_{i j}\right)$ is $I_{2}^{*}$ - Cauchy double sequence in $X$. Thus a double sequence $x=\left(x_{i j}\right)$ is $I_{2}$ - Cauchy double sequence in $X$ if and only if $x=\left(x_{i j}\right)$ is $I_{2}^{*}$ - Cauchy double sequence in $X$.

\section{References}

[1] G.Bachman, Introduction to p-Adic Numbers and Valuation Theory (Academic Press, 1964).

[2] G. Bachman and L. Narici, Functional Analysis (New York and London, Academic Press, 1966).

[3] Balakrishna Tripathy and Binod Chandra Tripathy, On I-convergent double sequences ,Soochow Journal of Mathematics, 31 , 2005 549-560,.

[4] P.Das ,P.Kostyrko,W.Wikzynski and P.Malik,I and I* -convergence of double sequences , Math. Slovaca, 58(5),2008,605-620.

[5] Erdinç Dündar and Bilal Altay, On Some Properties of $I_{2}-$ Convergence and $I_{2}-$ Cauchy of Double Sequences, Gen. Math. Notes, Vol. 7, No.1, November 2011, pp.1-12.

[6] Erdinç Dündar and Özer Talo, $I_{2}$ - Cauchy Double Sequences of Fuzzy Numbers, Gen. Math. Notes, 16(2), $2013,103-114$.

[7] S. Gähler, Linear 2-normerte raume, Math. Nachr, 28, 1965, 1-45.

[8] M. Gurdal, On ideal convergent sequences in 2-normed spaces, Thai Journal of Mathematics, 4(1), 2006, 85-91.

[9] M. Gurdal and Isil Acik, On I-Cauchy sequences in2-normedspaces,Mathematical Inequalities \& Applications, 11( 2) ,2008,349354.

[10] M. Gurdal, Ahmet Sahiner, Extremal I-Limit points of Double Sequences, Applied Mathematical E-Notes, 8,2008, 131-137.

[11] P. Kostyrko, M. Macaj and T. Salat, I-convergence, Real Anal. Exchange, 26(2), 2000, 669-686.

[12] P. Kostyrko, M. Macaj, T. Salat and M. Sleziak, I-convergence and extremal I-limit points, Math. Slovaca, 55, 2005, 443-464.

[13] Mehmet Acikgoz, N. Aslan, N. Koskeroglu and S. Araci, p-adic approach to linear 2-normed spaces, Mathematica Moravica, 13(2) 2009, 7-22.

[14] F.Nuray and W.H.Ruckle, Generalized statistical convergence and convergence free spaces, J.Math.Anal.Appl., 245,2000, 513-527.

[15] Pratulananda Das and Prasanta Malik, On Extrimal I-limit points of double sequences, Tatra Mt.Math.Publ. 40, 2008, 91-102.

[16] A.Pringsheim, Zur theorie der zweifach unendlichen Zahlenfolgen, Math. Ann., 53, 1900, $289-321$.

[17] W. Raymond, R. Freese and J. Cho, Geometry of Linear 2-Normed Spaces (Nova Science Publishers, 2001).

[18] Saeed Sarabadan,Fatemeh Amoee Arani and Siamak Khalehoghli, A Condition for the Equivalence of I and I*-convergence in 2normed spaces, Int.J.Contemp. Math. Sciences, 6(43), 2011, 2147-2159.

[19] Saeed Sarabadan and Sorayya Talebi, On I-convergence of Double sequences in 2- normed spaces, Int.J.Contemp.Math.Sciences,.7(14), 2012, 673-684.

[20] A.Sahiner, M. Gurdal, S. Saltan and H. Gunawan, Ideal convergence in 2-normed spaces, Taiwanese Journal of Mathematics, 11(5) 2007, 1477-1484. 
[21] B.Surender Reddy, Equivalence of p-adic 2-norms in p-adic linear 2-normed spaces, International Journal of Open Problems in Computer Science and Mathematics, 3(5),(2010, 25-38.

[22] B.Surender Reddy and D.Shankaraiah, On Ideal convergent sequences in p-adic linear 2- normed spaces, General Mathematics Notes17(1), 2013, 88-104.

[23] B.Surender Reddy and D.Shankaraiah, On I-Cauchy sequences in p-adic linear 2-normed spaces, International Journal of Pure and Applied Mathematics, 89(4), 2013, 483-496.

[24] B.Surender Reddy and D.Shankaraiah, On Ideal Convergent of Double Sequences in p-adic linear 2-normed spaces, Global Journal of Pure and Applied Mathematics, 10(1), 2014, 7-20.

[25] Vijay Kumar, On I and I*-Convergence of double sequences, Mathematical Communications 12, 2007, 171-181 